\title{
Cirrhosis Risk Score of the Donor Organ Predicts Early Fibrosis Progression after Liver Transplantation
}

\author{
Anca Zimmermann ${ }^{1}$, Felix Darstein ${ }^{1,2}$, Maria Hoppe-Lotichius ${ }^{3}$, Gerrit Toenges ${ }^{4}$, Anja Lautem ${ }^{3}$, Frédéric Abel ${ }^{1}$, \\ Arno Schad ${ }^{5}$, Jens Mittler ${ }^{2,3}$, Johanna Vollmar ${ }^{1}$, Daniel Grimm ${ }^{1}$, Hauke Lang ${ }^{2,3}$, Peter R. Galle ${ }^{1,2}$, Tim Zimmermann ${ }^{1,2 *}$, \\ Detlef Schuppan $^{6,7^{*}}$
}

1) $1^{\text {st }}$ Department of Medicine,

Cirrhosis Center Mainz

(CCM), University Medical

Center Mainz, Germany

2) Interdisciplinary Transplant

Center, University Medical

Center Mainz, Germany

3) Department of General-,

Visceral- and Transplantation-

Surgery, University Medical

Center Mainz, Germany

4) Institute of Medical

Biostatistics, Epidemiology

and Informatics, University

Medical Center, Mainz,

Germany

5) Institute of Pathology,

University Medical Center,

Mainz, Germany

6) Institute of Translational Immunology, Liver Fibrosis Center, University Medical Center Mainz, Germany

7) Division of

Gastroenterology, Beth Israel

Deaconess Medical Center,

Harvard Medical School,

Boston, MA, USA

Address for correspondence: Anca Zimmermann, M.D. 1st Department of Medicine, University Medical Center Mainz

Langenbeckstr. 1, 55131

Mainz, Germany

zimmeran@uni-mainz.de

Received: 03.11.2018

Accepted: 20.12.2018

${ }^{*}$ Contributed equally

\section{ABSTRACT}

Background \& Aims: Fibrosis progression (FP) after liver transplantation (LT) increases morbidity and mortality. Biomarkers are needed for early prediction of FP. A recipient's seven-gene cirrhosis risk score (CRS) has been associated with FP, especially in non-transplant cohorts. A broader validation of CRS, including the genotype of the donor-organ and HCV-negative patients is lacking. We therefore analyzed the impact of donor- and recipient-specific genotypes on FP after LT in a large cohort of HCV-positive and -negative patients. Method: Genotyping from liver biopsies ( $\mathrm{n}=201$ donors) and peripheral blood ( $\mathrm{n}=442$ recipients) was performed. Cirrhosis risk score was correlated with FP at 1 and 5 years after LT.

Results: Fibrosis $\geq \mathrm{F} 2$ was documented in $26.5 \%$ of the recipients' CRS group (R-CRS) (defined by recipient's genotype) and in $23.4 \%$ of the donors' CRS- group (D-CRS) (defined by donor's genotype). Cumulative incidence for fibrosis $\geq F 2$ was higher in patients with D-CRS $>0.7(\mathrm{p}=0.03)$. While the R-CRS showed no prognostic relevance, $\mathrm{D}$-CRS $>0.7$ was associated with higher hazard ratios (HRs) for fibrosis $\geq \mathrm{F} 2$ (HR=2.04; $\mathrm{p}=0.01)$, especially in HCV-negative patients $(\mathrm{HR}=2.59, \mathrm{p}=0.03)$. Donors' CRS $>0.7$ was associated with higher risk for $\geq F 2$ in 1 -year protocol biopsies $(\mathrm{p}<0.001)$. Among the patients in whom both the recipient's and donor's CRS were available, fibrosis $\geq \mathrm{F} 2$ was encountered more frequently in patients with a $\mathrm{D}$-CRS $>0.7$, in combination with any R-CRS, compared to patients with D-CRS scores $\leq 0.7$ ( $p=0.034$ ). Donors' AZIN1, STXBP5L, TRPM5 genotypes carried a higher risk for fibrosis $\geq \mathrm{F} 2$ in subgroups.

Conclusion: High D-CRS $>0.7$ predicted early FP after LT, especially in HCV negative patients.

Key words: liver transplantation - fibrosis - cirrhosis risk score.

Abbreviations: CRS: cirrhosis risk score; D: donor; FP: fibrosis progression; HBV: hepatitis B virus; HCC: hepatocellular carcinoma; HCV: hepatitis C virus; HR: hazard ratio; LT: liver transplantation; $\mathrm{N}$ fib: number of patients with fibrosis $\geq F$; R: recipient; RC: recurrent cirrhosis.

\section{INTRODUCTION}

Liver transplantation (LT) is the last therapeutic option for patients with end-stage liver disease. The development of LT into a successful therapy over the last few decades has resulted in improved postoperative short- and long-term survival. Therefore, management of comorbidities and prevention of recurrence of the underlying disease has become increasingly important, especially in view of the donor shortage [1]. Accelerated fibrosis progression
(FP) and recurrent cirrhosis (RC) have emerged as challenging complications after LT [2-8].

Today, in the new era of highly effective antiviral therapies, posttransplant recurrence of viral hepatitis C (HCV) has developed into a manageable condition, eliminating many cases of post-transplant cirrhosis. However, alcohol relapse, non-alcoholic steatohepatitis (NASH), autoimmune liver diseases and other underlying diseases can lead to rapid posttransplant FP and RC [3-6].

Several donor and recipient dependent clinical risk factors are known to be associated with FP, especially in patients with recurrent hepatitis C [9]. Immunosuppression and increasing donor age are held responsible for an accelerated rate of FP after LT [9-11]. These clinical risk factors can only in part explain why some patients develop very fast RC, within a few years after LT, while others show no significant fibrosis at the 5-year protocol biopsy [12]. Beside factors related to the 
transplant procedure or the immunosuppressive management, genetic factors of the donor or recipient appear to influence FP after LT [13].

Few data regarding the effect of genetic predisposition for FP exist in post-transplant patients. Layden et al. [14] identified significant multivariate associations of FP in post-transplant $\mathrm{HCV}$-infected patients with recipient gene variants of the interleukin 28B (IL28B), DEAD box protein 5 (DDX5), patatinlike phospholipase domain containing 3 (PNPLA3), suppressor of cytokine signaling-3 (SOCS3) and malectin (MLEC) genes [14].

A seven-gene signature, identified by genome wide association studies, is associated with FP in HCV-infected patients prior to LT, as reported by Huang et al. [15]. These genetic polymorphisms were used to establish a cirrhosis risk score (CRS). Patients with a CRS $<0.5$ were categorized as at low risk for FP; CRS values between 0.5 and 0.7 were associated with an intermediate risk and a CRS $>0.7$ was predictive of a high FP risk [15]. Importantly, several of the CRS genes have been confirmed functionally to be involved in hepatic fibrogenesis $[16,17]$.

Overall, 75\% of the HCV-reinfected patients after LT displaying a high CRS $>0.7$ developed at least F2 fibrosis during follow-up, independently of known clinical risk factors such as donor age, sex of the recipient or acute rejection [18]. These data suggest that the genetic signature of the recipient predicts the likelihood of severe liver fibrosis development in the graft after HCV recurrence. However, the effect of the genetic risk profile of the donor organ on FP after LT as well the role of the recipient's CRS in patients with underlying liver diseases other than hepatitis $\mathrm{C}$ have not been investigated.

We therefore analyzed for the first time the relevance of the CRS for the development of fibrosis after LT in a large cohort of HCV-positive and HVC-negative LT recipients, including the impact of the donor genotypes.

\section{MATERIAL AND METHOD}

\section{Patients}

We evaluated 611 patients with LTs performed between 1998 and 2012 at the Interdisciplinary Transplant Center, University Medical Center Mainz. Inclusion criteria were: liver transplanted patients older than 18 years, Caucasians, complete histological data and genotyping available. Exclusion criteria were: retransplantation, successfully treated $\mathrm{HCV}$ infection after LT, fibrosing cholestatic hepatitis $\mathrm{C}$, ischemic type biliary lesions, postoperative bile duct and vascular complications. Data was obtained from prospectively performed protocol liver biopsies and from prospectively maintained transplant databases. The selection process is shown in Fig. 1. Patients with $\mathrm{HCV}$, hepatitis B viral (HBV) infection, hepatocellular carcinoma (HCC), occurred in the context of an underlying liver disease, alcoholic cirrhosis and other underlying diseases for LT (NASH, primary biliary cholangiopathy, primary sclerosing cholangitis, autoimmune hepatitis) were included.

All HCV-positive patients developed HCV-reinfection post-LT, diagnosed by positive HCV-RNA and characteristic laboratory findings. Alcoholic etiology before LT or alcohol relapse after LT were defined as alcohol intake $>30 \mathrm{~g} /$ day for men and $>20 \mathrm{~g} /$ day for women.

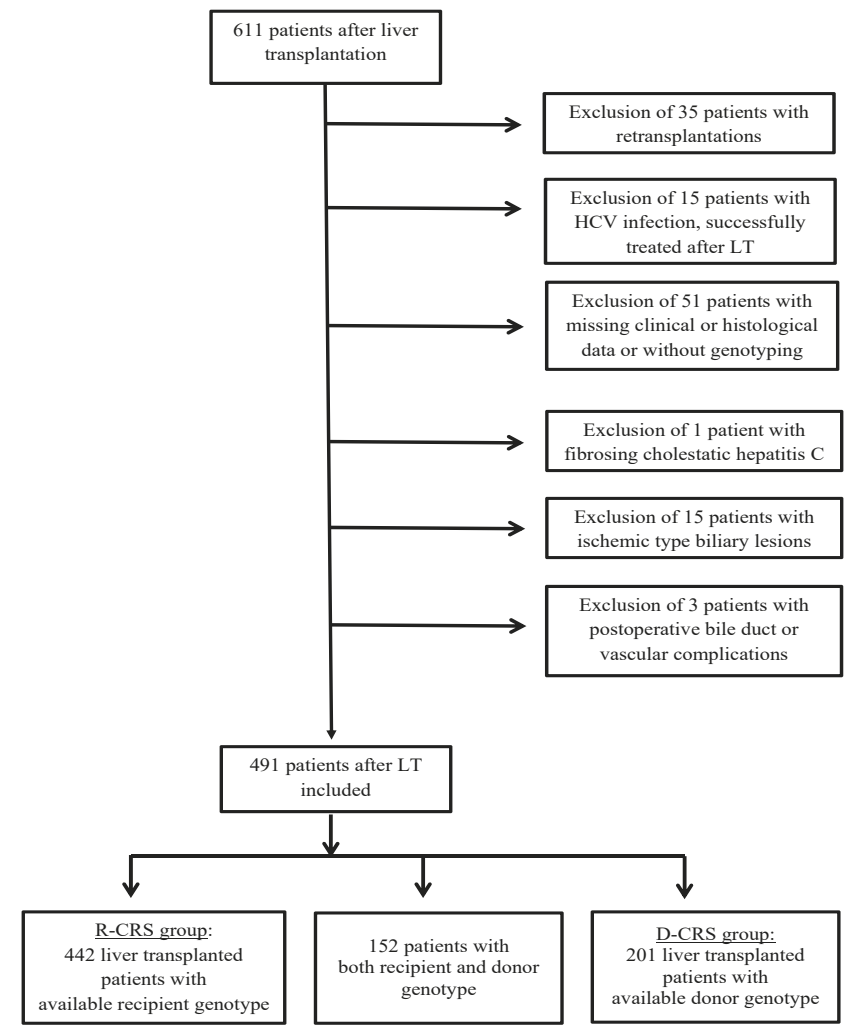

Fig. 1. Selection process.

Patients' data were provided by electronic medical records and patient charts. Medical history, data of physical examination and biochemical blood tests were obtained at least every 3-6 months within the first 5 years after LT, depending on the clinical status. Further data regarding donors' and recipients' demographics, patients' comorbidities, surgical complications and survival were recorded.

Finally, 491 patients met the inclusion criteria and were genotyped, after written informed consent. Our study followed the ethical guidelines of the Declaration of Helsinki and was approved by the local Ethics Committee of the State of Rhineland-Palatinate [no. 837.533.11(8075)].

\section{Histological Assessment}

Liver biopsies were performed in all donor livers before reperfusion and graft fibrosis $>$ F1 before LT was excluded. Protocol liver biopsies were prospectively performed at 1 and 5 years after LT by Menghini needle, if patients gave their informed approval. In cases of abnormal laboratory values or clinical findings, an additional histological evaluation of the transplanted liver was performed. Liver specimens were assessed by two experienced pathologists. The second one was blinded to the results of the first pathologist and both were blinded to the patients' medical history. Staging of liver fibrosis was performed according to the scoring system of Desmet and Scheuer [19], using a scale from F0-F4. Significant fibrosis progression was assumed when a difference of at least one fibrosis stage to $\geq \mathrm{F} 2$ occurred between the biopsy at baseline (time of transplantation) and at follow-up. Cumulative incidences of fibrosis were defined as the probability to develop significant fibrosis $(\geq F 2)$, proven by biopsy during the time of observation. 


\section{Immunosuppression}

Patients received immunosuppression according to individual risk factors and comorbidities. The standard immunosuppressive regimen was a combination of a calcineurin inhibitor with mycophenolate mofetil. Target trough levels for tacrolimus were $5-7 \mathrm{ng} / \mathrm{ml}$ during the first 5 years and 3-5 ng/ml thereafter. For cyclosporine, the target trough level was 70-90 ng/ml during the first 5 years and 50 $70 \mathrm{ng} / \mathrm{ml}$ thereafter. All patients received steroids in the first 3-6 months post LT.

\section{Genotyping}

Samples used for DNA analysis were collected prospectively. Genomic DNA from the recipient was extracted from $200 \mu \mathrm{l}$ EDTA-blood using the QIAamp DNA Blood Mini Kit (Qiagen, Hilden, Germany) according to the manufacturer's protocol. Donor DNA was extracted from pre-implantation liver biopsies (QIAamp DNA Mini Kit; Qiagen, Hilden, Germany). Determination of the genotypes of the following genes established in the CRS [15], was performed by real time PCR on a LightCycler ${ }^{\circledR} 2.0$ device (Roche, Mannheim, Germany) using a commercial LightSNiP (SimpleProbe) assay purchased from Tib-MolBiol (Berlin, Germany, https://www.tib-molbiol. de) according to the manufacturer's recommendations: adaptor-related protein complex 3 S2 (AP3S2) (rs2290351), aquaporin 2 (AQP2) (rs2878771), antizyme inhibitor 1 (AZIN1) (rs62522600), degenerative spermatocyte homolog 1 lipid desaturase (DEGS1-NVL) (rs4290029), syntaxin binding protein 5-like (STXBP5L) (rs17740066), toll-like receptor 4 (TLR4) (rs4986791) and transient receptor potential cation channel M5 (TRPM5) (rs 886277).

Samples were set up in a final volume of $10 \mu \mathrm{l}$, containing $1 \mu \mathrm{l}$ of DNA solution ( 50 ng), $1 \mu \mathrm{l}$ of LightCycler FastStart DNA Master HybProbe Mix (Roche Molecular Biochemicals, Mannheim, Germany), $0.8 \mu \mathrm{l}$ of $\mathrm{MgCl} 2(25 \mathrm{mM})$, and $0.5 \mu \mathrm{l}$ of LightSNiP reagent mix (Tib-MolBiol, Berlin, Germany. Cycling conditions were as follows: initial denaturation $\left(10 \mathrm{~min} 95^{\circ} \mathrm{C}\right)$ followed by 45 cycles of denaturation $\left(10 \mathrm{~s}\right.$ at $\left.95^{\circ} \mathrm{C}\right)$, annealing $\left(10 \mathrm{~s}\right.$ at $\left.60^{\circ} \mathrm{C}\right)$, and elongation $\left(15 \mathrm{~s}\right.$ at $\left.72^{\circ} \mathrm{C}\right)$. Subsequently, DNA melting was performed: $20 \mathrm{~s}$ at $95^{\circ}, 20 \mathrm{~s}$ at $40^{\circ} \mathrm{C}$ followed by continuous heating to $85^{\circ} \mathrm{C}$ (ramp rate $0.2^{\circ} \mathrm{C} / \mathrm{s}$ ). Thereafter, samples were cooled down to $40^{\circ} \mathrm{C}$ (ramp rate $20^{\circ} \mathrm{C} / \mathrm{s}$ ). When the probe melted off the template, fluorescence resonance energy transfer no longer took place and fluorescence was converted to melting peaks using software that plotted the negative derivative of fluorescence with respect to temperature.

\section{CRS Algorithm}

The CRS was calculated as described based on the SNPs of the 7 target genes (AP3S2, AQP2, AZIN1, DEGS1, STXBP5L, TLR4 and TRPM5) using a naïve Bayes formula [15].

We stratified the patients into three CRS categories: $<0.5$, $0.5-0.7$ and $>0.7$. To increase the statistical power, CRS-values $<0.5$ and $0.5-0.7$ were pooled. Associations of CRS with clinical variables, histology and risk factors for fibrosis were analysed.

\section{Statistical Analysis}

All statistical analyses were performed using $\mathrm{R}$ version 3.4.2 [20]. Quantitative data are expressed as medians with interquartile ranges. Categorical variables are given as frequencies and percentages, respectively, and for the comparison of two or more groups Fisher's exact test or a chi-square test was applied. Between-group differences for quantitative variables were assessed using the Wilcoxon rank sum test.

Cumulative incidences for fibrosis were calculated under consideration of death and retransplantation as competing risks using the cmprsk-package in $\mathrm{R}[21,22]$. Comparisons of cumulative incidence functions between subgroups were performed with special log-rank-tests that consider competing risks, again using the cmprsk-package [22]. Univariable and multivariable Cox proportional hazards models were used to evaluate the association of potential risk factors with the fibrosis risk in terms of cause-specific Hazard ratios (HR) for fibrosis. Thereby, the proportional hazards assumption was evaluated using Schoenfeld residuals.

All tests were performed two-sided. Our complete data analysis is exploratory. Hence no adjustments for multiple testing were performed. For all tests we used a 0.05-level to define statistically relevant deviations from the respective null hypotheses. However, due to the large number of tests, p-values should be interpreted with caution and in connection with effect estimates.

\section{RESULTS}

From all the 491 patients, patients were chosen according to genotype availability. The recipient's genotype was available in 442 patients (R-CRS group). In 201 patients, donor genotypes were available (D-CRS group). The two patient groups overlapped in 152 patients, in which both recipients and donors' genotypes were known (Fig. 1). Demographic characteristics are presented in Table I for the two groups, defined according to the available genotype. Mean follow-up was 5.5 (3.2-9.4) years for the R-CRS group and 5.8 (2.7-8.3) years for the D-CRS group. Overall, 62\% $(n=274)$ of the recipients had a CRS $\leq 0.7$ [25.6\% $(\mathrm{n}=113)$ with a CRS $<0.5$, $36.4 \%(n=161)$ between 0.5 and 0.7$]$, and $38 \%(n=168)$ of patients had a CRS score $>0.7$. Among patients with known donor genotype, $60.2 \%(n=121)$ of the patients had a CRS score $\leq 0.7[23.8 \%(\mathrm{n}=48)<0.5,36.4 \%(\mathrm{n}=73)$ between $0.5-0.7)]$ and $39.8 \%(n=80)$ had a CRS $>0.7$. For HCV positive patients in the R-CRS group, the median CRS was 0.59 (interquartile range 0.41-0.75) and for HCV-negative recipients in this group it was $0.61(0.51-0.77), \mathrm{p}=0.156$; for $\mathrm{HCV}$ positive patients in the D-CRS group it was $0.63(0.52-0.77)$ and for $\mathrm{HCV}$ negative patients in the same group $0.61(0.46-0.77), \mathrm{p}=0.295$.

\section{Cumulative incidences of fibrosis and CRS}

Development of fibrosis $\geq \mathrm{F} 2$ was documented in 117 (26.5\%) patients from the R-CRS group and in 47 (23.4\%) patients from the D-CRS group during follow-up. The prevalence of fibrosis $\geq F$ 2 differed according to the underlying liver disease. For example in the R-CRS group transplanted for HCC, we found significantly fewer patients with fibrosis $\geq$ F2 (32/167) compared to patients transplanted for other underlying liver diseases (85/275), $\mathrm{p}=0.006$. Cumulative incidences for fibrosis $\geq \mathrm{F} 2$ were higher in recipients with a D-CRS $>0.7$ in the whole 
Table I. Patients' demographics

\begin{tabular}{|c|c|c|}
\hline & $\mathrm{R}-\mathrm{CRS}^{1}(\mathrm{n}=442)$ & $\mathrm{D}-\mathrm{CRS}^{2}(\mathrm{n}=201)$ \\
\hline \multicolumn{3}{|l|}{ Gender } \\
\hline Male (n; \%) & $300(67.8)$ & $141(70.1)$ \\
\hline Female (n; \%) & $142(32.2)$ & $60(29.9)$ \\
\hline \multicolumn{3}{|l|}{ Age (years) } \\
\hline Median (quartiles) & $55.1(49.0-61.7)$ & $56.3(50.2-63.9)$ \\
\hline \multicolumn{3}{|l|}{$\operatorname{BMI}^{3}\left(\mathrm{~kg} / \mathbf{m}^{2}\right)$} \\
\hline Median (quartiles) & $24.3(16.2-43.5)$ & $23.8(15.3-44.8)$ \\
\hline \multicolumn{3}{|l|}{$\mathrm{HCV}^{4}(\mathrm{n} ; \%)$} \\
\hline negative & $333(75.3)$ & $144(71.6)$ \\
\hline Male & $225(67.5)$ & $100(69.4)$ \\
\hline Female & $108(32.4)$ & $44(30.5)$ \\
\hline Age (years; median, quartiles) & $55.8(48.6-61.6)$ & $57.9(51.5-64.1)$ \\
\hline CRS $<0.5 ; 0.5-0.7 ;>0.7$ & 77 (23.1); 125 (37.5); $131(39.3)$ & $38(26.4) ; 52(36.1) ; 54(37.5)$ \\
\hline positive & $109(24.6)$ & $57(28.3)$ \\
\hline Male & $65(59.6)$ & $41(71.9)$ \\
\hline Female & $44(40.4)$ & $16(28.1)$ \\
\hline Age (years; median, quartiles) & $53.7(49.1-62.3)$ & $53.1(49.3-59.8)$ \\
\hline CRS $<0.5 ; 0.5-0.7 ;>0.7$ & $36(33.0) ; 36(33.1) ; 37(33.9)$ & $10(17.5) ; 21(36.9) ; 26(45.6)$ \\
\hline \multicolumn{3}{|l|}{$\operatorname{HBV}^{5}$ (n; \%) } \\
\hline negative & $376(85.1)$ & $167(83.1)$ \\
\hline positive & $66(14.9)$ & $34(16.9)$ \\
\hline \multicolumn{3}{|l|}{$\mathrm{HCC}^{6}(\mathrm{n} ; \%)$} \\
\hline negative & $275(62.2)$ & $109(54.2)$ \\
\hline positive & $167(37.8)$ & $92(45.8)$ \\
\hline \multicolumn{3}{|l|}{ Alcohol (n; \%) } \\
\hline no & $241(54.5)$ & $119(59.2)$ \\
\hline yes & $201(45.5)$ & $82(40.8)$ \\
\hline \multicolumn{3}{|l|}{ Others (n; \%) } \\
\hline total & $66(14.9)$ & $28(13.9)$ \\
\hline $\mathrm{NASH}^{7}(\mathrm{n} ; \%)$ & $29(43.9)$ & $14(50)$ \\
\hline $\mathrm{PBC}^{8}(\mathrm{n} ; \%)$ & $16(24.2)$ & $1(3.5)$ \\
\hline $\operatorname{PSC}^{9}(\mathrm{n} ; \%)$ & $15(22.7)$ & $5(17.9)$ \\
\hline Autoimmune (n; \%) & $6(9.0)$ & $8(28.6)$ \\
\hline CRS (median; quartiles) & $0.61(0.48-0.77)$ & $0.62(0.50-0.77)$ \\
\hline$<0.5$ & $113(25.6)$ & $48(23.8)$ \\
\hline $0.5-0.7$ & $161(36.4)$ & $73(36.4)$ \\
\hline$>0.7$ & $168(38.0)$ & $80(39.8)$ \\
\hline \multicolumn{3}{|l|}{ Donor characteristics } \\
\hline \multirow[t]{3}{*}{ Donor age (years; median, quartiles) } & $50.1(39-62)-\mathrm{R}-\mathrm{CRS}$ total & $50.9(40-65)-$ D-CRS total \\
\hline & 50.4 (38-63)- R-CRS, HCV positive & 50.9 (42-62.5)- D-CRS, HCV positive \\
\hline & 50.0 (40-62)- R-CRS, HCV negative & 51 (40-65)- D-CRS, HCV negative \\
\hline \multirow[t]{3}{*}{ Donor sex (males/females) } & 224/218- R-CRS total & 95/106- D-CRS total \\
\hline & 57/52- R-CRS, HCV positive & 26/31- D-CRS, HCV positive \\
\hline & 167/166- R-CRS, HCV negative & 69/75- D-CRS, HCV negative \\
\hline
\end{tabular}

CRS: cirrhosis risk score; ${ }^{1}$ R-CRS: LT patients with available recipients' genotypes, ${ }^{2}$ D-CRS: LT patients with available donors' genotypes; ${ }^{3} \mathrm{BMI}$ - body mass index; ${ }^{4} \mathrm{HCV}$ - hepatitis $\mathrm{C}$ virus; ${ }^{5} \mathrm{HBV}$ - hepatitis B virus; ${ }^{6} \mathrm{HCC}$ - hepatocellular carcinoma; ${ }^{7} \mathrm{NASH}$ - non-alcoholic steatohepatitis; ${ }^{8} \mathrm{PBC}$ - primary biliary cholangiopathy; ${ }^{9} \mathrm{PSC}$ - primary sclerosing cholangitis 
D-CRS group $(\mathrm{p}=0.03)$ and by trend in the corresponding HCV-negative subgroup $(\mathrm{p}=0.06)$, compared to patients with a CRS $\leq 0.7$ (Fig. 2A, B). There was no impact of the donor genotype in $\mathrm{HCV}$-positive patients $(\mathrm{p}=0.47$ ) (Fig. 2C) and no relevant difference in the cumulative incidence for fibrosis $\geq \mathrm{F} 2$ between patients stratified according to the R-CRS, in the whole R-CRS group ( $p=0.892)$, in the HCV-positive $(p=0.3)$ and HCV-negative R-CRS subgroup ( $\mathrm{p}=0.73$ ).
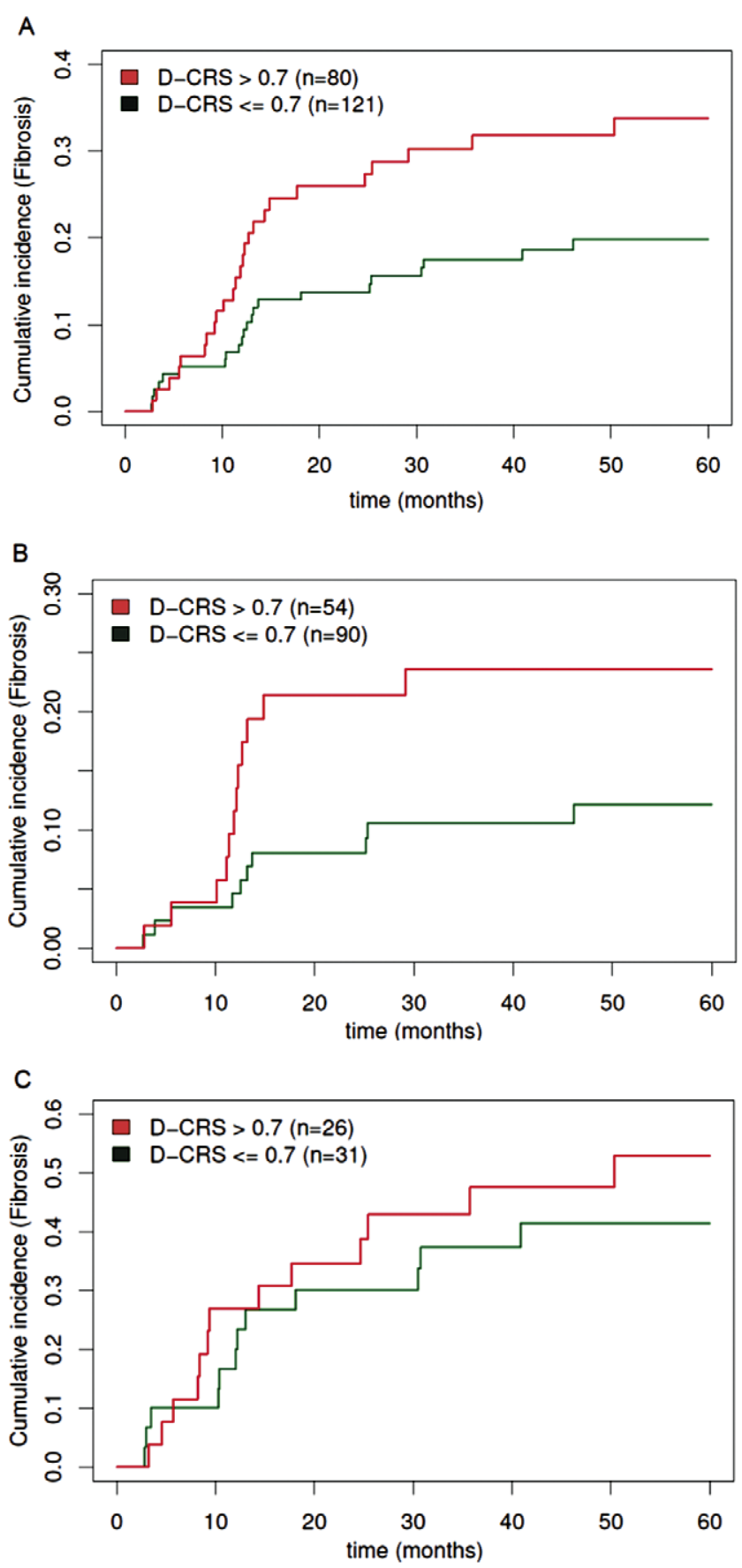

Fig. 2. Cumulative incidences of fibrosis $\geq F 2$ in patients stratified according to the Donor (D)- CRS: A: in the whole D-CRS group (80 patients with D-CRS $>0.7 ; 121$ patients with $\mathrm{D}$-CRS $\leq 0.7 ; \mathrm{p}=0.03$ ); $\mathrm{B}$ : in the HCV-negative D-CRS subgroup (54 patients with D-CRS $>0.7$; 90 patients with $\mathrm{D}$-CRS $\leq 0.7 ; \mathrm{p}=0.06) \mathrm{C}$ : in the $\mathrm{HCV}$-positive D-CRS subgroup (26 patients with $\mathrm{D}$-CRS $>0.7 ; 31$ patients with D-CRS $\leq 0.7 ; \mathrm{p}=0.47$ )
The influence of different potential risk factors for fibrosis was analyzed by univariable Cox proportional hazard models (Table II). As expected, the strongest predictive factor for the development of fibrosis $\geq \mathrm{F} 2$ was HCV-infection $(\mathrm{p}<0.001)$. Further influencing factors were $\mathrm{HBV}$ infection in the R-CRS group, HCC in the HCV-negative R-CRS subgroup and recipient's age in the $\mathrm{HCV}$ positive R-CRS and D-CRS subgroups. Donor's CRS $>0.7$ was associated with higher hazard ratios (HRs) for fibrosis $\geq \mathrm{F} 2(\mathrm{p}=0.01)$ in the whole $\mathrm{D}$-CRS group and in HCV-negative patients $(\mathrm{p}=0.03)$.

Table II. Predictive factors for fibrosis $\geq F 2$ (univariable analysis by the Cox-PH-model)

\begin{tabular}{|c|c|c|}
\hline Predictive factor & $\mathrm{HR}^{1}(95 \% \mathrm{CI})$ & $\mathrm{p}^{2}$ \\
\hline R-CRS ${ }^{3}$ & $\mathrm{n}=442, \mathrm{n} \mathrm{fib}^{4}=117$ & \\
\hline Sex male (ref. female) & $0.96(0.65-1.41)$ & 0.855 \\
\hline Recipient age & $0.98(0.97-1.01)$ & 0.123 \\
\hline Donor age & $1.00(0.99,1.02)$ & 0.113 \\
\hline $\mathrm{HBV}^{5}$ positive (ref. negative) & $0.34(0.17-0.70)$ & 0.003 \\
\hline $\mathrm{HCV}^{6}$ positive (ref. negative) & $3.31(2.29-4.76)$ & $<0.001$ \\
\hline $\mathrm{HCC}^{7}$ positive (ref. negative) & $0.77(0.53-1.14)$ & 0.204 \\
\hline Alcohol yes (ref. no) & $0.76(0.51-1.11)$ & 0.163 \\
\hline R-CRS-groups $>0.7($ ref. $\leq 0.7)$ & $0.98(0.67-1.43)$ & 0.938 \\
\hline R-CRS, HCV neg & $\mathrm{n}=333, \mathrm{n}$ fib $=55$ & \\
\hline Sex male (ref. female) & $0.67(0.40,1.11)$ & 0.120 \\
\hline Recipient age & $0.99(0.97,1.01)$ & 0.345 \\
\hline Donor age & $1.01(0.99,1.02)$ & 0.226 \\
\hline HBV positive (ref. negative) & $0.49(0.28,1.09)$ & 0.083 \\
\hline HCC positive (ref. negative) & $0.54(0.30,0.99)$ & 0.046 \\
\hline Alcohol yes (ref. no) & $0.90(0.55,1.549)$ & 0.545 \\
\hline R-CRS-groups $>0.7($ ref. $\leq 0.7)$ & $0.93(0.56,1.57)$ & 0.812 \\
\hline R-CRS, HCV pos & $\mathrm{n}=109 ; \mathrm{n}$ fib $=62$ & \\
\hline Sex male (ref. female) & $1.65(0.90,3.03)$ & 0.104 \\
\hline Recipient age & $0.96(0.92,0.99)$ & 0.008 \\
\hline Donor age & $1.01(0.99,1.03)$ & 0.341 \\
\hline HBV positive (ref. negative) & $0.24(0.03,1.71)$ & 0.153 \\
\hline HCC positive (ref. negative) & $0.75(0.44-1.28)$ & 0.295 \\
\hline Alcohol yes (ref. no) & $1.26(0.66,2.38)$ & 0.482 \\
\hline R-CRS-groups $>0.7($ ref. $\leq 0.7)$ & $1.27(0.73,2.20)$ & 0.398 \\
\hline $\mathrm{D}-\mathrm{CRS}^{8}$ & $\mathrm{n}=201, \mathrm{n}$ fib $=47$ & \\
\hline Sex male (ref. female) & $0.81(0.44,1.49)$ & 0.517 \\
\hline Recipient age & $0.99(0.97,1.01)$ & 0.567 \\
\hline Donor age & $1.01(0.99,1.03)$ & 1.170 \\
\hline HBV positive (ref. negative) & $0.54(0.21,1.38)$ & 0.203 \\
\hline HCV positive (ref. negative) & $3.37(1.90,6.00)$ & $<0.001$ \\
\hline HCC positive (ref. negative) & $1.10(0.63,1.95)$ & 0.735 \\
\hline Alcohol yes (ref. no) & $0.77(0.42,1.43)$ & 0.424 \\
\hline D-CRS-groups $>0.7($ ref. $\leq 0.7)$ & $2.04(1.15,3.63)$ & 0.014 \\
\hline D-CRS, HCV neg & $\mathrm{n}=144, \mathrm{n}$ fib $=22$ & \\
\hline Sex male (ref. female) & $0.58(0.24,1.9)$ & 0.208 \\
\hline Recipient age & $1.02(0.99,1.07)$ & 0.960 \\
\hline Donor age & $1.01(0.98,1.03)$ & 0.258 \\
\hline
\end{tabular}




\begin{tabular}{lll}
\hline Table II (continued) & & \\
\hline HBV positive (ref. negative) & $0.86(0.29,2.54)$ & 0.788 \\
HCC positive (ref. negative) & $1.58(0.68,3.67)$ & 0.280 \\
Alcohol yes (ref. no) & $1.17(0.50,2.71)$ & 0.703 \\
D-CRS-groups $>0.7$ (ref. $\leq 0.7)$ & $2.59(1.12,6.01)$ & $\mathbf{0 . 0 2 5}$ \\
\hline D-CRS, HCV pos & $\mathrm{n}=57, \mathrm{n} \mathrm{fib}=25$ & \\
\hline Sex male (ref. female) & $1.19(0.49,2.85)$ & 0.69 \\
Recipient age & $0.94(0.90,0.99)$ & $\mathbf{0 . 0 1 5}$ \\
Donor age & $1.01(0.98,1.04)$ & 0.251 \\
HBV positive (ref. negative) & $0.42(0.05,3.10)$ & 0.394 \\
HCC positive (ref. negative) & $0.49(0.21,1.07)$ & 0.072 \\
Alcohol yes (ref. no) & $1.08(0.37,3.16)$ & 0.880 \\
D-CRS-groups $>0.7$ (ref. $\leq 0.7)$ & $1.17(0.53,2.56)$ & 0.699 \\
\hline
\end{tabular}

${ }^{1} \mathrm{HR}$ - hazard ratio; ${ }^{2} \mathrm{p}-\mathrm{p}$ value; ${ }^{3} \mathrm{R}$-CRS - LT patients with available recipients' genotypes; ${ }^{4} \mathrm{n}$ fib - number of fibrosis, ${ }^{5} \mathrm{HBV}$ - hepatitis B virus; ${ }^{6} \mathrm{HCV}$ - hepatitis C virus; ${ }^{7} \mathrm{HCC}$ - hepatocellular carcinoma; ${ }^{8} \mathrm{D}$-CRS: LT patients with available donors' genotypes.

To evaluate confounder-adjusted effect estimates for CRS, we performed further analyses using multivariable Cox proportional hazard models (Table III). For analysis of the HCV-negative D-CRS subgroup, we decided to include stepwise only two potential confounders in the multivariable analysis, due to the limited number of cases with relevant fibrosis in this subgroup $(n=22)$. When evaluating this specific subgroup with different models including two further factors besides CRS, we observed in all combinations (7 models tested) that a D-CRS $>0.7$ was strongly associated with fibrosis $\geq \mathrm{F} 2$ compared to genotype scores $\leq 0.7(\mathrm{p}<0.05)$.

\section{Early fibrosis and CRS}

To investigate the association between CRS and early FP $(\geq F 2)$, the available 1-year protocol biopsies after LT were analyzed. Interestingly, a donor's $C R S>0.7$ was more frequently encountered in patients with $\geq \mathrm{F} 2$ in the 1-year protocol biopsy after $\mathrm{LT}$, than in patients with F0 and F1. This was observed for the whole D-CRS group $(\mathrm{p}<0.001)$, as well as for the HCV-negative subgroup $(p<0.001)$, while in the HCV-positive subgroup no effect of the donors' genotype was evidenced ( $\mathrm{p}=0.40$ ), (Fig. $3 \mathrm{~A}, \mathrm{~B}, \mathrm{C}$ ).

Among the patients in whom both the recipient's and donor's CRS were available, 94 patients agreed to protocol biopsy after one year. Fibrosis $\geq \mathrm{F} 2$ was encountered more frequently in patients with a $\mathrm{D}$-CRS $>0.7$, in combination with any R-CRS (11/34), compared to patients with D-CRS scores $\leq 0.7$, combined with any R-CRS $(8 / 60)(\mathrm{p}=0.034)$.

\section{Severe fibrosis and CRS}

With a limited number of recurrent cirrhosis, a R-CRS $>0.7$ was not predictive for the development of advanced fibrosis $(\geq \mathrm{F} 3)$ at 1 and 5 years after LT, neither in the whole, nor in the HCV-positive or HCV-negative R-CRS subgroup. However, a D-CRS $>0.7$ in HCV-negative patients showed a tendency towards a more frequent association with $\mathrm{F} 3 / \mathrm{F} 4$ than a CRS value $\leq 0.7$ ( $\mathrm{p}=0.06)$ (Table IV, Supplementary material).

\section{Individual SNPs and fibrosis $\geq \mathbf{F} 2$}

The influence of individual SNPs on the development of fibrosis $\geq \mathrm{F} 2$ was analyzed by the Cox-proportional hazard
Table III. Predictive factors for fibrosis $\geq F 2$ (multivariable analysis by the Cox-PH-model for CRS and potential confounders)

\begin{tabular}{|c|c|c|}
\hline Group/Variables & $\mathrm{HR}^{1}(95 \% \mathrm{CI})$ & $\mathrm{p}^{2}$ \\
\hline \multicolumn{3}{|l|}{$R-\mathrm{CRS}^{3}(\mathrm{n}=442, \mathrm{n}$ fib $=117)$} \\
\hline Sex male (ref. female) & $1.11(0.72-1.67)$ & 0.595 \\
\hline Recipient age & $0.97(0.95-0.99)$ & 0.033 \\
\hline Donor age & $1.01(0.99-1.02)$ & 0.053 \\
\hline $\mathrm{HBV}^{4}$ positive (ref. negative) & $0.42(0.19-0.89)$ & 0.023 \\
\hline $\mathrm{HCV}^{5}$ positive (ref. negative) & $3.35(2.25-5.01)$ & $<0.001$ \\
\hline $\mathrm{HCC}^{6}$ positive (ref. negative) & $0.74(0.49-1.13)$ & 0.169 \\
\hline Alcohol yes (ref. no) & $0.89(0.58-1.38)$ & 0.627 \\
\hline R-CRS-groups $>0.7$ (vs. $\leq 0.7$ ) & $1.09(0.75-1.60)$ & 0.629 \\
\hline \multicolumn{3}{|l|}{$\mathrm{D}-\mathrm{CRS}^{7}(\mathrm{n}=201, \mathrm{n}$ fib $=47)$} \\
\hline Sex male (ref. female) & $0.72(0.36-1.38)$ & 0.330 \\
\hline Recipient age & $0.98(0.95-1.02)$ & 0.389 \\
\hline Donor age & $1.01(0.99-1.03)$ & 0.126 \\
\hline HBV positive (ref. negative) & $0.78(0.29-2.05)$ & 0.612 \\
\hline HCV positive (ref. negative) & $3.30(1.71-6.35)$ & $<0.001$ \\
\hline HCC positive (ref. negative) & $0.97(0.51-1.83)$ & 0.937 \\
\hline Alcohol yes (ref. no) & $1.19(0.58-2.43)$ & 0.630 \\
\hline D-CRS-groups $>0.7$ (vs. $\leq 0.7$ ) & $1.69(0.93-3.06)$ & 0.079 \\
\hline \multicolumn{3}{|c|}{ D-CRS, HCV negative $(\mathrm{n}=144, \mathrm{n}$ fib $=22)$} \\
\hline \multicolumn{3}{|l|}{ Model 1 of multivariable analysis } \\
\hline Donor age & $1.01(0.98-1.03)$ & 0.368 \\
\hline HBV positive (ref. negative) & $0.99(0.33-2.99)$ & 0.999 \\
\hline D-CRS-groups >0.7 (vs. $\leq 0.7$ ) & $2.48(1.05-5.83)$ & 0.037 \\
\hline \multicolumn{3}{|l|}{ Model 2 of multivariable analysis } \\
\hline Donor age & $1.01(0.98-1.03)$ & 0.407 \\
\hline Alcohol yes (ref. no) & $1.10(0.46-2.59)$ & 0.823 \\
\hline D-CRS-groups >0.7 (vs. $\leq 0.7$ ) & $2.48(1.06-5.79)$ & 0.034 \\
\hline \multicolumn{3}{|l|}{ Model 3 of multivariable analysis } \\
\hline Donor age & $1.01(0.98-1.03)$ & 0.368 \\
\hline HCC positive (ref. negative) & $1.79(0.78-4.19)$ & 0.768 \\
\hline D-CRS-groups >0.7 (vs. $\leq 0.7$ ) & $2.70(1.15-6.33)$ & 0.021 \\
\hline \multicolumn{3}{|l|}{ Model 4 of multivariable analysis } \\
\hline Donor age & $1.01(0.99-1.04)$ & 0.536 \\
\hline Recipient age & $1.02(0.98-1.07)$ & 0.284 \\
\hline D-CRS-groups >0.7 (vs. $\leq 0.7$ ) & $2.46(1.05-5.74)$ & 0.036 \\
\hline \multicolumn{3}{|l|}{ Model 5 of multivariable analysis } \\
\hline Donor age & $1.01(0.99-1.03)$ & 0.306 \\
\hline Sex male (ref. female) & $0.56(0.24-1.33)$ & 0.194 \\
\hline D-CRS-groups >0.7 (vs. $\leq 0.7$ ) & $2.42(1.04-5.65)$ & 0.039 \\
\hline \multicolumn{3}{|l|}{ Model 6 of multivariable analysis } \\
\hline HCC positive (ref. negative) & $1.76(0.74-4.18)$ & 0.195 \\
\hline Alcohol yes (ref. no) & $1.06(0.45-2.51)$ & 0.876 \\
\hline D-CRS-groups >0.7 (vs. $\leq 0.7$ ) & $2.78(1.19-6.49)$ & 0.017 \\
\hline \multicolumn{3}{|l|}{ Model 7 of multivariable analysis } \\
\hline HBV positive (ref. negative) & $1.09(0.34-3.49)$ & 0.881 \\
\hline Alcohol yes (ref. no) & $1.22(0.50-2.98)$ & 0.658 \\
\hline D-CRS-groups $>0.7$ (vs. $\leq 0.7)$ & $2.62(1.12-6.14)$ & 0.025 \\
\hline
\end{tabular}

${ }^{1} \mathrm{HR}$ - hazard ratio; ${ }^{2} \mathrm{p}$ - $\mathrm{p}$ value; ${ }^{3} \mathrm{R}$-CRS: LT patients with available recipients' genotypes; ${ }^{4} \mathrm{HBV}$ - hepatitis B virus; ${ }^{5} \mathrm{HCV}$ - hepatitis C virus; ${ }^{6} \mathrm{HCC}$ hepatocellular carcinoma; ${ }^{7}$ D-CRS: LT patients with available donors' genotypes. 
model, in an explorative manner. No individual SNP showed a HR significantly different from 1 , neither in the R-CRS nor in the D-CRS group. However, the donor's AZIN1 genotype (A/G
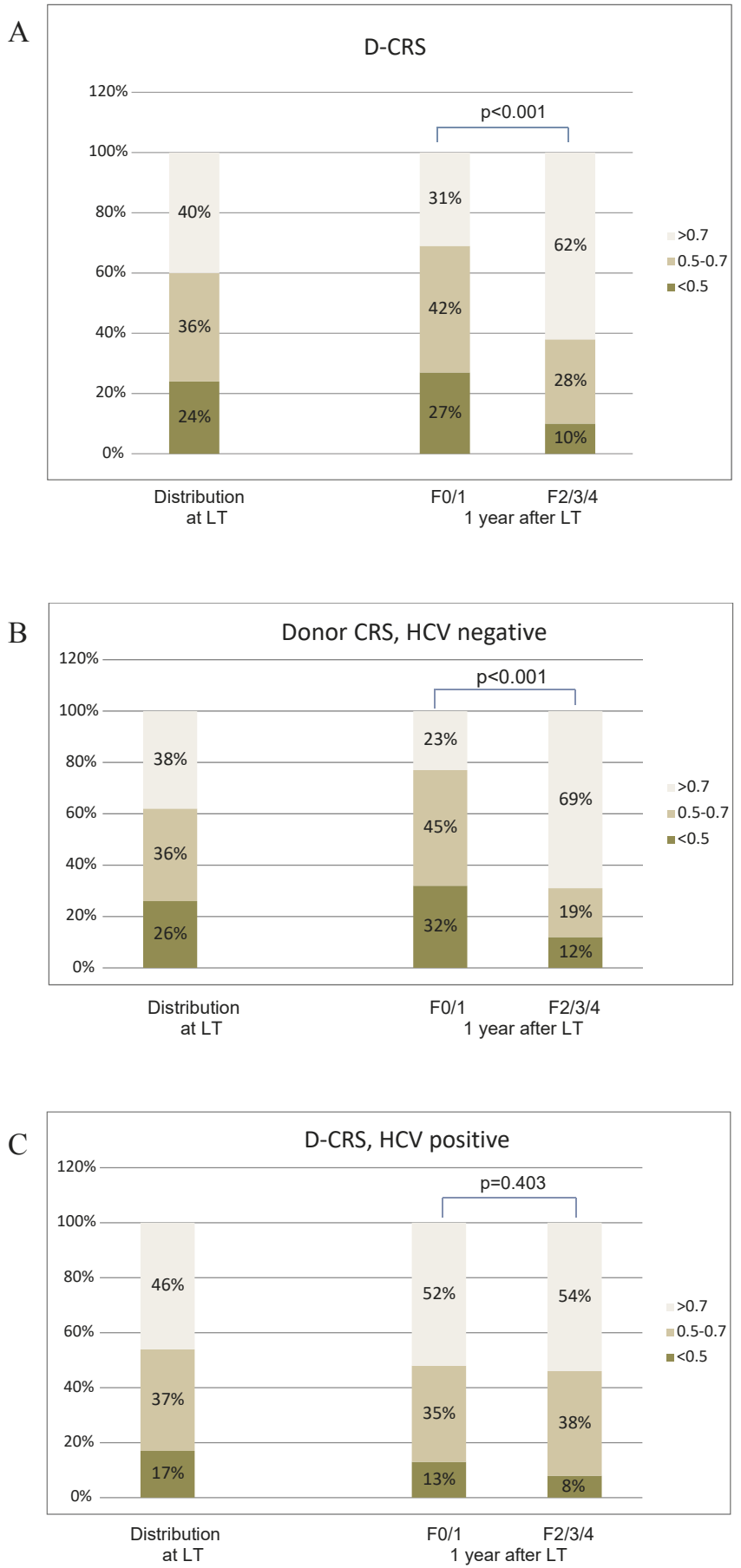

Fig. 3. Fibrosis ( $\geq \mathrm{F} 2$ compared to $<\mathrm{F} 2$ ) in the protocol biopsy one year after LT according to the D-CRS. A: all D-CRS patients, $\mathrm{n}=121$ protocol biopsies at 1 year, $\mathrm{n}=92$ with $<\mathrm{F} 2,29$ with $\geq \mathrm{F} 2$. B: HCVnegative patients of the $\mathrm{D}$-CRS group, $\mathrm{n}=85$ protocol biopsies at 1 year, $\mathrm{n}=69$ with $<\mathrm{F} 2,16$ with $\geq \mathrm{F} 2$. C: HCV-positive patients of the D-CRS group, $\mathrm{n}=36$ protocol biopsies at 1 year, $\mathrm{n}=23$ with $<\mathrm{F} 2, \mathrm{n}=13$ with $\geq \mathrm{F} 2$. Patients receiving a donor liver with a CRS $>0.7$ showed a higher risk for significant early fibrosis $\geq F 2$ than with a CRS $\leq 0.7$ in the whole group and in the HCV-negative subgroup. D-CRS - patients in whom the donor genotype was available; $\mathrm{HCV}$ - hepatitis C virus; LT - liver transplantation. vs. $G / G$ ) was associated with a relevant risk for fibrosis $\geq F 2$ in the group of HCV-positive recipients (HR 4.01; 95\% CI: 1.978.16; $\mathrm{p}=0.001$ ). Furthermore, the donor's STXBP5L genotype carried a higher risk for fibrosis $\geq \mathrm{F} 2$ in $\mathrm{HCV}$-negative patients $(\mathrm{HR}=2.72 ; 95 \% \mathrm{CI}=1.22-6.14) ; \mathrm{p}=0.02)$. A similar effect was shown for the TRPM5 genotype in HCV-positive patients of the D-CRS subgroup ( $\mathrm{HR}=0.52 ; 95 \% \mathrm{CI}=0.29-0.93$; $\mathrm{p}=0.03$ ).

\section{DISCUSSION}

Recurrence of fibrosis is an important cause of morbidity and mortality in LT-recipients [9]. Clinical risk factors for FP have been previously identified [12]. In the past few years several studies about the association of genetic factors with progression of fibrosis have been published [23-26].

Three retrospective longitudinal cohort studies evaluated the CRS as a predictive factor for FP in HCV positive patients [18, 27, 28]. Marcolongo et al. [27] analyzed a cohort of 271 Caucasian patients with mild chronic hepatitis $C$ who underwent a diagnostic biopsy, and received a follow-up biopsy 5 years later. Only patients with F0-F2 at first biopsy were included and none of these patients received antiviral therapy during the study interval. Mean CRS was higher in patients with FP than in those without. Later, Trepo et al. [28] confirmed these results in a smaller, restrospective analysis of 56 Caucasian patients with chronic hepatitis C before LT, with F0-F1 at the first biopsy, and follow up after 5 years. The median CRS was higher in patients with fibrosis progression $\geq$ F2. In the logistic regression model, only a CRS $>0.7$ had a significant influence on FP [28]. Do O et al. [18] assessed the predictive value of CRS after LT and evaluated 137 patients transplanted for $\mathrm{HCV}$-induced cirrhosis, with the exclusion of $\mathrm{HBV}$-coinfection or alcohol abuse. A CRS $>0.7$ was predictive for the development of F2 or F3 fibrosis in the protocol biopsies after one, three and five years. Moreover, the interval up to the development of fibrosis $\geq \mathrm{F} 3$ was shorter with a CRS $>0.7$ [18].

Our study went beyond these reports. First, it is the largest analysis on the prognostic relevance of CRS after LT to date. Second, in view of HCV-infection per se is a strong and independent predictor of fibrosis (with a HR $>3$ in our patients), we also included HCV-negative patients in this analysis. Third, in all previous studies, the CRS was only determined in the transplant recipients, while the donor liver CRS likely has a prominent association with the risk of posttransplant FP. There is no previous report of the impact of D-CRS on severity of fibrosis after LT and only few donor genotypes are known to affect FP after LT [29]. We therefore determined for the first time the D-CRS to correlate it with graft fibrosis development.

When analysing the effect of the D-CRS according to HCV status, we found a significant association between $\geq \mathrm{F} 2$ and the donor's CRS $>0.7$ in HCV-negative patients ( $p=0.03$ ). In addition, cumulative incidences for fibrosis $\geq \mathrm{F} 2$ were higher in recipients with a donor CRS $>0.7$ in the whole $\mathrm{D}$-CRS group $(p=0.03)$ and by trend in the corresponding HCV-negative subgroup $(\mathrm{p}=0.06)$. The 1 -year protocol biopsy showed a significant association of early fibrosis $\geq F 2$ with a D-CRS $>0.7$ in all patients of the D-CRS group $(p<0.001)$ and in the HCVnegative $\mathrm{D}$-CRS patients $(\mathrm{p}<0.001)$. In contrast to do $\mathrm{O}$ et al. 
[18], we did not find an association of the R-CRS scores $>0.7$ with higher cumulative incidences for fibrosis $\geq \mathrm{F} 2$ in biopsies.

There are some differences between our study and the previous publications in patients' selection, endpoint definition and data analysis [15, 18, 27, 28]. Huang et al. [15] performed a cross-sectional study that did not stratify patients according to the duration of $\mathrm{HCV}$-infection and that did not address current dynamics of FP. The probability for development of severe fibrosis stage $\geq \mathrm{F} 3$ depends on the duration of infection and prior "second hits" other than the genetic risk that may not be active at the time of analysis [30]. Patients with F1 and F2 fibrosis were excluded and the prevalence of fibrosis $\geq F 3$ was $90.9 \%$ in the validation cohort and $62 \%$ in the training cohort [15].

The other three studies had a longitudinal retrospective design and allow a better comparison to our study. However, the endpoints differed in all cohorts.

The study by do $\mathrm{O}$ et al. [18] shows the strongest similarity to our study. Although patient groups seemed comparable with respect to recipient's age, men were more prevalent in their than in our HCV-positive R-CRS subgroup (63.5\% vs. 59.6\%), while our donors were older (median 50.4 years in our cohort vs. 42 years). Severe fibrosis ( $\geq$ F3) one year after LT was lower in our patients with $6.3 \%(28 / 442)$ in the total R-CRS group and $10 \%(11 / 109)$ in the HCV-positive R-CRS subgroup vs. $18.3 \%$ $(21 / 115)$ in the HCV-positive patients reported by do O et al. [18]. High CRS $>0.7$ scores were more frequent in their than in our cohort $(46.7 \%$ in the HCV positive cohort of do $\mathrm{O}$ et al. vs. $38 \%$ in our whole R-CRS group and $34 \%$ in our HCV positive R-CRS subgroup). These differences between the two studies regarding the cohorts of $\mathrm{HCV}$-positive LT recipients might explain why do $\mathrm{O}$ et al. [18] found an association of the recipient's CRS with fibrosis after LT, whereas this could not have been confirmed by us.

A limitation of our analysis using the CRS is that only some SNPs of the donor could be relevant for FP after LT, whereas others could influence fibrosis when belonging to the recipient. In the current era of safe and effective direct acting-antivirals, there is no relevant role for CRS in HCV-positive patients. However, the current functional studies related to the CRSSNPs suggest fibrogenic potential independent of the cause of the liver disease $[16,17]$ and we found an effect of the donor's genotype mainly in $\mathrm{HCV}$-negative patients.

Since fibrosis is the result of a complex interplay between donor and recipient features, considering recipient or donor variables alone might lead to an incomplete analysis, which is the major limitation of our study. The two only in part overlapping groups in our study could have been built up retrospectively only by random, to the extent to which genotyping was available in this big real life cohort. Furthermore, we cannot provide longitudinal data for serological or ultrasound-based surrogate markers for fibrosis, since these methods were not extensively available at the beginning of the study. In our statistical analyses, the limited number of events with respect to the number of covariates may lead to some degree of overfitting. On the other hand, to prevent biased estimates, presumably important covariates should not be omitted in multivariable survival models.

\section{CONCLUSIONS}

Our data provide first evidence that the CRS genotype of the donor organ is associated with early fibrosis progression after LT, especially in HCV-negative patients. Further validation of the CRS and related scores based on donor and recipient genetic factors in well-defined prospective cohorts are warranted to assess FP and individual risk for recurrent cirrhosis and to improve therapeutic options for patients after LT.

Conflicts of interest: There is no conflict of interest. No funding sources were involved in the study design, in the collection, analysis and interpretation of data, in the writing or the decision to submit the article for publication.

Author contributions: A.Z., T.Z., F.D., D.S. designed the study, collected and analysed data, and wrote the paper. F.D., D.G., J.M., J.V. collected and analysed data, made critical revision. T.Z., D.S., P.R.G., H.L. analysed and interpreted data, and made critical revision. M.H.L., G.T. collected and analysed data, performed statistical analysis, drafting. F.A. and A.L. collected and analysed data, performed DNA extraction and genotyping, critical revision. A.S. performed histological evaluation of fibrosis, acquisition of data, critical revision. All authors approved the final version.

Acknowledgements: This work was supported by an intramural grant of the University of Mainz to T.Z. (Inneruniversitäre Forschungsförderung) and by an ERC Advanced Grant (Fibroimaging) to D.S.

We thank Larissa Herbel, 1st Department of Medicine and Uli Suessdorf, Department of General, Visceral and Transplantation Surgery, University of Mainz for excellent technical assistance with the patients' samples.

Supplementary material: To access the supplementary material visit the online version of the J Gastrointestin Liver Dis at http://dx.doi. org/10.15403/jgld.2014.1121.281.crr

\section{REFERENCES}

1. Adam R, Karam V, Delvart V, et al. Evolution of indications and results of liver transplantation in Europe. A report from the European Liver Transplant Registry (ELTR). J Hepatol 2012;57:675-688. doi:10.1016/j. jhep.2012.04.015

2. Levy G, Villamil FG, Nevens F, et al. REFINE: a randomized trial comprising cyclosporine $\mathrm{A}$ and tacrolimus on fibrosis after liver transplantation for hepatitis C. Am J Transplant 2014;14:635-646. doi:10.1111/ajt.12620

3. Rice JP, Eickhoff J, Agni R, Ghufran A, Brahmbhatt R, Lucey MR. Abusive drinking after liver transplantation is associated with allograft loss and advanced fibrosis. Liver Transpl 2013;19:1377-1386. doi:10.1002/1t.23762

4. Fosby B, Karlsen TH, Melum E. Recurrence and rejection in liver transplantation for primary sclerosing cholangitis. World J Gastroenterol 2012;18:1-15. doi:10.3748/wjg.v18.i1.1

5. Hytoroglou P, Gutierrez GA, Freni M, et al. Recurrence of primary biliary cirrhosis and development of autoimmune hepatitis after liver 
transplant: A blind histologic study. Hepatol Res 2009;39:577-584 doi:10.1111/j.1872-034X.2008.00483.x

6. Miyagawa-Hayashino A, Haga H, Egawa H, Hayashino Y, Uemoto S, Manabe T. Idiopathic post-transplantation hepatitis following living donor liver transplantation, and significance of autoantibody titre for outcome. Transpl Int 2009;22:303-312. doi:10.1111/j.14322277.2008.00803.x

7. Nagai S, Yoshida A, Kohno K, et al. Peritransplant absolute lymphocyte count as a predictive factor for advanced recurrence of hepatitis $\mathrm{C}$ after liver transplantation. Hepatology 2014;59:35-45. doi:10.1002/hep.26536

8. Angulo P, Kleiner DE, Dam-Larsen S, et al. Liver fibrosis, but no other histologic features, is associated with long-term outcomes of patients with nonalcoholic fatty liver disease. Gastroenterology 2015;149:389397. doi:10.1053/j.gastro.2015.04.043

9. Berenguer M. Outcome of posttransplantation hepatitis $\mathrm{C}$ virus disease - is it the host, the virus, or how we modify the host and/or the virus? Liver Transpl 2002;8:889-891. doi:10.1053/jts.2002.35841

10. Machicao VI, Bonatti H, Krishna M, et al. Donor age affects fibrosis progression and graft survival after liver transplantation for hepatitis $\mathrm{C}$. Transplantation 2004;77:84-92. doi:10.1097/01.TP.0000095896.07048.BB

11. Selzner N, Girgrah N, Lilly L, et al. The difference in the fibrosis progression of recurrent hepatitis $\mathrm{C}$ after live donor liver transplantation versus deceased donor liver transplantation is attributable to the difference in donor age. Liver Transpl 2008;14:1778-1786. doi:10.1002/ lt.21598

12. Zimmermann T, Otto C, Hoppe-Lotichus M, et al. Risk factors in patients with rapid recurrent hepatitis $\mathrm{C}$ virus-related cirrhosis within 1 year after liver transplantation. Transplant Proc 2009;41:2549-2556. doi:10.1016/j.transproceed.2009.06.120

13. Eurich D, Neumann UP, Boas-Knoop S, et al. YKL-40 gene polymorphism affects acute cellular rejection and fibrosis progression after transplantation for hepatitis $C$ virus-induced liver disease. J Gastroenterol Hepatol 2013;28:153-160. doi:10.1111/j.14401746.2012.07270.x

14. Layden JE, Tayo BO, Cotler SJ, et al. Association of genetic variants with rapid fibrosis: progression after liver transplantation for hepatitis C. Transplantation 2014;97:1072-1078. doi:10.1097/01. TP.0000440953.06886.a3

15. Huang H, Shiffman ML, Friedman S, et al. A 7 gene signature identifies the risk of developing cirrhosis in patients with chronic hepatitis C. Hepatology 2007;46:297-306. doi:10.1002/hep.21695

16. Paris AJ, Snapir Z, Christopherson CD, et al. A polymorphism that delays fibrosis in hepatitis $\mathrm{C}$ promotes alternative splicing of AZIN1, reducing fibrogenesis. Hepatology 2011;54:2198-2207. doi:10.1002/hep.24608

17. Guo J, Loke J, Zheng F, et al. Functional linkage of cirrhosis-predictive single nucleotide polymorphisms of Toll-like receptor 4 to hepatic stellate cell responses. Hepatology 2009;49:960-968. doi:10.1002/ hep. 22697

18. do O NT, Eurich D, Schmitz P, et al. A 7-gene signature of the recipient predicts the progression of fibrosis after liver transplantation for hepatitis C virus infection. Liver Transpl 2012;18:298-304. doi:10.1002/ 1t.22475

19. Desmet VJ, Gerber M, Hoofnagle JH, Mans M, Scheuer PJ. Classification of chronic hepatitis: diagnosis, grading and staging. Hepatology 1994;19:1513-1520. doi:10.1002/hep.1840190629

20. R Core Team. R: A language and environment for statistical computing. R Foundation for Statistical Computing 2017, Vienna, Austria. Available from: https://www.R-project.org/

21. Putter H, Fiocco M, Geskus RB. Tutorial in biostatistics: competing risks and multi-state models. Statist Med 2007;26:2389-2430.

22. Bob Gray. cmprsk: Subdistribution Analysis of Competing Risks. R package version 2.2-7, 2014. Available from: https://CRAN.R-project. org $/$ package $=$ cmprsk

23. Eurich D, Bahro M, Boas-Knoop S, et al. Transforming growth factor betal polymorphisms and progression of graft fibrosis after liver transplantation for hepatitis $\mathrm{C}$ virus--induced liver disease. Liver Transpl 2011;17:279-288. doi:10.1002/lt.22190

24. Eurich D, Boas-Knoop S, Struecker B, Neuhaus R, Neuhaus P, Bahra M. Genetic variants of STAT- 4 affect the development of graft fibrosis after liver transplantation for HCV-induced liver disease. Transplantation 2013:95:203-208. doi:10.1097/TP.0b013e318277e2f6

25. Ge D, Fellay J, Thompson AJ, et al. Genetic variation in IL28B predicts hepatitis C treatment-induced viral clearance. Nature 2009;461:399-401 doi:10.1038/nature08309

26. Zimmermann T, Hoppe-Lotichus M, Körner A, et al. The recipient CYP2D6 allele 4-associated poor metabolizer status correlates with an early fibrosis development after liver transplantation. Transpl Int 2011;24:1059-1067. doi:10.1111/j.1432-2277.2011.01305.x

27. Marcolongo M, Young B, Dal Pero F, et al. A seven-gene signature (cirrhosis risk score) predicts liver fibrosis progression in patients with initially mild chronic hepatitis C. Hepatology 2009;50:1038-1044. doi:10.1002/hep.23111

28. Trepo E, Potthoff A, Pradat P, et al. Role of a cirrhosis risk score for the early prediction of fibrosis progression in hepatitis $\mathrm{C}$ patients with minimal liver disease. J Hepatol 2011;55:38-44. doi:10.1016/j. jhep.2010.10.018

29. Dunn W, O’Neil M, Zhao J, et al. Donor PNPLA3 rs738409 genotype affects fibrosis progression in liver transplantation for hepatitis C. Hepatology 2014;59:453-460. doi:10.1002/hep.26758

30. Berenguer M, Schuppan D. Progression of liver fibrosis in posttransplant hepatitis C: mechanisms, assessment and treatment. J Hepatol 2013;58:1028-1041. doi:10.1016/j.jhep.2012.12.014 
Table IV (Supplementary data). Biopsy-data, at 1 and 5 years after the LT, according to the recipient's (R-) and the donor's (D-) CRS. Comparison between patients without fibrosis (F0) and those with severe fibrosis $\geq \mathbf{F 3}$ ( $\mathrm{p}$ values were calculated with the Fisher's exact test).

\begin{tabular}{|c|c|c|c|c|}
\hline Biopsy data at follow-up time points & 1 year & \multicolumn{3}{|c|}{5 years } \\
\hline Fibrosis stage (Desmet and Scheuer) & 0 & $3 / 4$ & 0 & $3 / 4$ \\
\hline$R$-CRS ${ }^{1}, H C V^{2}$ positive & $\mathrm{n}$ & $\mathrm{n}$ & $\mathrm{n}$ & $\mathrm{n}$ \\
\hline CRS $<0.5$ & 11 & 3 & 7 & 3 \\
\hline $0.5-0.7$ & 12 & 2 & 4 & 3 \\
\hline$>0.7$ & 8 & 6 & 6 & 4 \\
\hline $\mathrm{p}^{3}(\mathrm{CRS} \leq 0.7 ; \mathrm{CRS}>0.7)$ & \multicolumn{2}{|c|}{0.135} & \multicolumn{2}{|c|}{1.00} \\
\hline R-CRS, HCV negative & $\mathrm{n}$ & $\mathrm{n}$ & $\mathrm{n}$ & $\mathrm{n}$ \\
\hline $\mathrm{CRS}<0.5$ & 23 & 4 & 21 & 10 \\
\hline $0.5-0.7$ & 51 & 7 & 41 & 16 \\
\hline$>0.7$ & 49 & 6 & 31 & 23 \\
\hline $\mathrm{p}(\mathrm{CRS} \leq 0.7 ; \mathrm{CRS}>0.7)$ & \multicolumn{2}{|c|}{0.796} & \multicolumn{2}{|c|}{0.145} \\
\hline$R-C R S$, total & $\mathrm{n}$ & $\mathrm{n}$ & $\mathrm{n}$ & $\mathrm{n}$ \\
\hline CRS $<0.5$ & 34 & 7 & 28 & 13 \\
\hline $0.5-0.7$ & 63 & 9 & 45 & 19 \\
\hline$>0.7$ & 57 & 12 & 37 & 27 \\
\hline $\mathrm{p}(\mathrm{CRS} \leq 0.7 ; \mathrm{CRS}>0.7)$ & \multicolumn{2}{|c|}{0.672} & \multicolumn{2}{|c|}{0.136} \\
\hline D-CRS ${ }^{4}, H C V$ positive & $\mathrm{n}$ & $\mathrm{n}$ & $\mathrm{n}$ & $\mathrm{n}$ \\
\hline CRS $<0.5$ & 3 & 0 & 0 & 0 \\
\hline $0.5-0.7$ & 9 & 2 & 0 & 2 \\
\hline$>0.7$ & 12 & 2 & 0 & 0 \\
\hline $\mathrm{p}(\mathrm{CRS} \leq 0.7 ; \mathrm{CRS}>0.7)$ & \multicolumn{2}{|c|}{1.00} & \multicolumn{2}{|c|}{0.99} \\
\hline D-CRS, HCV negative & $\mathrm{n}$ & $\mathrm{n}$ & $\mathrm{n}$ & $\mathrm{n}$ \\
\hline $\mathrm{CRS}<0.5$ & 22 & 2 & 7 & 0 \\
\hline $0.5-0.7$ & 30 & 1 & 7 & 0 \\
\hline$>0.7$ & 15 & 4 & 9 & 2 \\
\hline $\mathrm{p}(\mathrm{CRS} \leq 0.7 ; \mathrm{CRS}>0.7)$ & \multicolumn{2}{|c|}{0.06} & \multicolumn{2}{|c|}{0.18} \\
\hline D-CRS, total & $\mathrm{n}$ & $\mathrm{n}$ & $\mathrm{n}$ & $\mathrm{n}$ \\
\hline CRS $<0.5$ & 25 & 2 & 7 & 0 \\
\hline $0.5-0.7$ & 39 & 3 & 7 & 2 \\
\hline$>0.7$ & 27 & 6 & 9 & 2 \\
\hline $\mathrm{p}(\mathrm{CRS} \leq 0.7 ; \mathrm{CRS}>0.7)$ & \multicolumn{2}{|c|}{0.17} & \multicolumn{2}{|c|}{0.99} \\
\hline
\end{tabular}

